\title{
Translational accuracy of a tethered ribosome
}

\author{
Celine Fabret and Olivier Namy ${ }^{\circledR *}$
}

Université Paris-Saclay, CEA, CNRS, Institute for Integrative Biology of the Cell (I2BC), 91198 Gif-sur-Yvette, France

Received January 07, 2021; Revised March 27, 2021; Editorial Decision March 29, 2021; Accepted May 04, 2021

\begin{abstract}
Ribosomes are evolutionary conserved ribonucleoprotein complexes that function as two separate subunits in all kingdoms. During translation initiation, the two subunits assemble to form the mature ribosome, which is responsible for translating the messenger RNA. When the ribosome reaches a stop codon, release factors promote translation termination and peptide release, and recycling factors then dissociate the two subunits, ready for use in a new round of translation. A tethered ribosome, called Ribo-T, in which the two subunits are covalently linked to form a single entity, was recently described in Escherichia coli. A hybrid ribosomal RNA (rRNA) consisting of both the small and large subunit rRNA sequences was engineered. The ribosome with inseparable subunits generated in this way was shown to be functional and to sustain cell growth. Here, we investigated the translational properties of Ribo-T. We analyzed its behavior during amino acid misincorporation, -1 or +1 frameshifting, stop codon readthrough, and internal translation initiation. Our data indicate that covalent attachment of the two subunits modifies the properties of the ribosome, altering its ability to initiate and terminate translation correctly.
\end{abstract}

\section{INTRODUCTION}

Translation is the last step in gene expression, in which the coding sequence of the messenger RNA (mRNA) is translated into the amino-acid sequence of the corresponding protein. Ribosomes catalyze protein synthesis, a vital cellular activity. Translation is a highly dynamic process, with four major phases: initiation, elongation, termination and ribosome recycling. During each phase, ribosomes form transient complexes with auxiliary translation factors that facilitate protein synthesis (1).

In all kingdoms of life, ribosomes consist of two subunits. The 30S subunit contains the 16S rRNA and 21 ribosomal proteins responsible for decoding genetic sequences. The 16S rRNA is involved in recognition of the Shine-Dalgarno (SD) sequence or ribosome binding site of the mRNA when it emerges from the RNA polymerase. This sequence is complementary to the $3^{\prime}$ end of the $16 \mathrm{~S}$ ribosomal RNA [anti-Shine-Dalgarno (ASD) sequence]. The 50S subunit contains two rRNA molecules, the $5 \mathrm{~S}$ and 23S rRNAs, with 33 proteins. It is responsible for catalyzing peptide bond formation. These two subunits associate with each other during translation initiation, rotate during elongation, and dissociate after protein release. This organization is thought to be essential for biogenesis, successful protein synthesis and cell viability.

A ribosome with tethered subunits, in which the two subunits were covalently linked in a single entity, was recently engineered in Escherichia coli $(2,3)$. The hybrid ribosomal RNA (rRNA) consisted of the small and large subunit rRNA sequences, linked by short RNA linkers. The resulting ribosome, Ribo-T, with its inseparable subunits, was shown to be functional and able to sustain the cell growth, although doubling times were slower (107 min for Ribo-T, 35 min for wild-type ribosomes). The rate protein synthesis with Ribo-T catalysis was $50 \%$ that with wild-type ribosomes. The slow growth of Ribo-T cells was probably due to Ribo-T biogenesis being slower than the biogenesis of wild-type ribosomes, rather than impaired Ribo-T activity (3).

Cell viability depends on a balance between rapid protein synthesis and accurate decoding of the genetic information. Translational error rates remain low throughout all steps in the process (4). However, signals present in specific mRNAs at defined locations can program a high rate of errors (5). These recoding events can occur during translation elongation (frameshifting) or at the termination step (stop codon readthrough). During frameshifts, the ribosome is induced to shift to an alternative, overlapping reading frame, whereas, in stop codon readthrough, the specific context of the termination codon may promote decoding with a nearcognate tRNA rather than a release factor. These sequences have specific impacts on translation fidelity, and are therefore powerful tools for investigations of the translation accuracy of Ribo-T ribosomes.

In this study, we investigated the translational properties of Ribo-T ribosomes. Do these ribosomes have the same range of translational missense error rates as wild-type ribosomes? Do they maintain the correct reading frame as faithfully as wild-type ribosomes? Do they terminate translation accurately at stop codons? Do they translate messen-

\footnotetext{
${ }^{*}$ To whom correspondence should be addressed. Tel: +33 1698262 30; Email: Olivier.namy@i2bc.paris-saclay.fr 
ger RNA accurately? We investigated the behavior of Ribo$\mathrm{T}$ ribosomes on misreading of near-cognate codons, and with recoding signals instructing the ribosome to change translational reading frame (frameshifting) or to read stop codons as sense codons (stop codon readthrough). We also investigated the preferential mode of translation used by Ribo-T ribosomes to express adjacent open reading frames on the same mRNA, given that the two subunits were inseparable. Our data reveal an impairment of tethered ribosomes for +1 frameshifting and termination, but show that these ribosomes are otherwise as effective as wild-type ribosomes.

\section{MATERIALS AND METHODS}

\section{Escherichia coli strains}

The experiments were performed with the Escherichia coli BL21 (DE3) strain (Invitrogen), with and without the poRibo-T2 vector carrying the oRibo-T ribosomal DNA for the expression of oRibo-T ribosomes (2). The ASD sequence of the Ribo-T 16S rRNA was altered from the wildtype sequence (5'-UCACCUCCUUA-3') to an orthogonal sequence (5'-UCAUUGUGGUA-3') (6), resulting in the oRibo-T ribosomes.

\section{Plasmids}

We used the bacterial pCL99 dual reporter system. This plasmid carries the lacZ-luc fusion gene, encoding the $\beta$ galactosidase and luciferase enzymes, under the control of the T7 promoter, together with the streptomycin resistance gene, and the CloDF13-derived CDF replicon (7). The various target sequences tested for translation accuracy were inserted by oligo-cloning into the $\mathrm{MscI}$ restriction site of pCL99, which placed them at the junction between the $l a c Z$ and $l u c$ genes. The cloned double-stranded oligonucleotides were obtained by hybridizing the two complementary oligonucleotides in ligation buffer after heating for 5 min at $100^{\circ} \mathrm{C}$ and incubation at room temperature. In-frame controls were constructed for each target sequence. All constructs were verified by sequencing. The target sequences are listed in Table 1.

For creation of the pCL99_oRibo-T constructs, containing the lac $Z$ gene controlled by an orthogonal SD sequence (oSD), the wild-type SD sequence (AGGAGG) was mutated to an orthogonal sequence, CACCAC, recognized by the oRibo-T ribosomes (2). A PCR fragment encompassing the lac $Z \mathrm{SD}$ sequence from the $A c l \mathrm{I}$ to $F s p \mathrm{I}$ sites of the vector was generated by PCR with the mutated sequence. An upstream PCR fragment was amplified with the oligonucleotides $5^{\prime}$ CTCTGCGACATCGTATAACGTTACTGG-3' (AclI site underlined) and 5'-GACCGTAATCATGGTATA TTGTGGTGTTAAAGTTAAACAAAATTATTTC-3' (mutated oSD sequence underlined), and a downstream PCR fragment was amplified with the oligonucleotides 5'-GTTTAACTTTAACACCACAATATACCATGA TTACGGAC-3' (mutated oSD underlined) and 5'CGCCATTCAGGCTGCGCAACTGTTGGG-3' (FspI site underlined). These two PCR fragments were joined by PCR with equimolar amounts of the two fragments as the template and oligonucleotides containing the $A c l I$ and FspI sites. The resulting PCR fragment was digested with AclI and FspI and inserted into the pCL99 constructs predigested with the same enzymes. The replacement of the wild-type $\mathrm{SD}$ sequence with the mutated oSD sequence was verified by sequencing, for all pCL99_oRibo-T constructs.

For constructs with the intergenic sequence containing a stem-loop structure (SL), the 5'-GCGATATCCC GTGGAGGGGCGCGTGGTGGCGGTGCT-3' and the phosphorylated 5'-CACCACGCGCCCCTCCAC GGGATATCGC-3' oligonucleotides were hybridized, as were the phosphorylated 5'-GCAGCACCGCCACC ACGCGCCCCTCCACGGGATATCGCT-3' and 5'AGCGATATCCCGTGGAGGGGCGCGTGGTGG CGGTGCTGCAGCACCGC-3' oligonucleotides. These two pairs of annealed oligonucleotides were then ligated together to produce the $75 \mathrm{bp}$ fragment forming a -105 $\mathrm{kcal} / \mathrm{mol}$ stem-loop structure (from RNA folder WebServer). This fragment was inserted into the SmaI site of the pCL99_oRibo-T oSD without SL. The presence of the correct SL was verified by sequencing.

The constructs for misreading of the lysine 529 codon of the luciferase mRNA were obtained by directed mutagenesis of the pCL99 and pCL99_oRiboT carrying the lacZ-luc fusion gene (in-frame controls for readthrough). The AAA lysine codon was modified either in a TTT phenylalanine codon, a CAA glutamine codon, an AGA arginine codon, or an AAT asparagine codon. Two $0.9 \mathrm{~kb}$ and $0.15 \mathrm{~kb}$ PCR fragments carrying the mutated K529 codon were amplified, respectively, with the oligonucleotides 5'-CCTAAGGGTGTGGCCCTTCCGC-3' and with 5'-GGATCTCTCTGATTTTTCTTGCGTCG AGNNNTCCGGTAAGACCTTTCGGTACTTCG-

$3^{\prime}$ (mutated nucleotides underlined $\mathrm{NNN}=\mathrm{AAA}$ or TTG or TCT or ATT), or with 5'-CGAAGTACCG AAAGGTCTTACCGGANNNCTCGACGCAAGA AAAATCAGAGAGATCC-3' (mutated nucleotides underlined NNN $=$ TTT or CAA or AGA or AAT) and 5'-GGTTATGCTAGTTATTGCTCAGCGG-3'. The couples of corresponding fragments were joined by PCR, then the resulting $1 \mathrm{~kb}$ fragments were digested by SphI and HindIII, and finally they were cloned into the pCL99 and pCL99_oRiboT vectors to replace the SphI-HindIII wildtype AAA K529-encompassing fragment. All constructs were verified by sequencing.

\section{Quantification of translation fidelity}

Escherichia coli BL21 or BL21 poRIBO-T2 competent cells were transformed with the pCL99 or pCL99_oRibo-T constructs, respectively, carrying the various target sequences used to test the translation accuracy of ribosomes. The transformants were cultured overnight at $30^{\circ} \mathrm{C}$ in $500 \mu \mathrm{l}$ of Luria broth supplemented with the appropriate antibiotics $(50 \mu \mathrm{g} / \mathrm{ml}$ streptomycin, $100 \mu \mathrm{g} / \mathrm{ml}$ ampicillin). Cultures were centrifuged for $2 \mathrm{~min}$ at room temperature, and the cell pellets were resuspended in $100 \mu l$ of luc buffer ( 25 $\mathrm{mM}$ Tris-phosphate $\mathrm{pH} 7.8,8 \mathrm{mM} \mathrm{MgCl}_{2}, 1 \mathrm{mM}$ DTT, 1 mM EDTA, $1 \%$ Triton X-100, 0.1\% BSA, $15 \%$ glycerol and 
Table 1. Target sequences used in the study

\begin{tabular}{|c|c|}
\hline Name & Sequence \\
\hline IS911_if & $\begin{array}{l}\text { CAGTTCTCGATAATCGGGAAACTCAGAGCGCATTATCCT } \\
\text { GAAATGGAGAATGAAATATTAAACAAAGGCTACCGCGCTCTTGATGTCAGACTCCCTGAA } \\
\text { CAGTTCTCGATAATCGGGAAACTCAGAGCGCATTATCCT }\end{array}$ \\
\hline IS3 & $\begin{array}{l}\text { CGGGATGAAGAGCTGGCTATCCTCCAAAAGGCCGCGACATACTTCGCGAAGCGCCTGAAA } \\
\text { TGAAGTATGTCTTTATTGAAAAACATCAGGCTGAG }\end{array}$ \\
\hline IS3_if & $\begin{array}{l}\text { CGGGATGAAGAGCTGGCTATCCTCCACAAAGGCCGCGACATACTTCGCGAAGCGCCTGAA } \\
\text { ATGAAGTATGTCTTTATTGAAAAACATCAGGCT }\end{array}$ \\
\hline dnaX_if & $\begin{array}{l}\text { CAGGGAGCAACCAAAGCAAACAAAGAGTGTACCGGCAGCCGCTACCCGCGCGCGGCCG } \\
\text { GTGAA }\end{array}$ \\
\hline dnaXoSD & $\begin{array}{l}\text { CCACCACCAACCAAAGCAAAAAAGAGTGTACCGGCAGCCGCTACCCGCGCGCGGCCGG } \\
\text { TGAA }\end{array}$ \\
\hline dnaXoSD if & $\begin{array}{l}\text { CCACCACCAACCAAAGCAAACAAAGAGTGTACCGGCAGCCGCTACCCGCGCGCGGCCG } \\
\text { GTGAA }\end{array}$ \\
\hline prfB & GTTCTTAGGGGGTATCTTTGACTACGAC \\
\hline TAG readthrough & ATGGGTGTCTAGGGCCCAGAG (corresponding constructs with TGA and TAA) \\
\hline wt SD & TAATAATAACCTGGGCAGGCCATGTCTGCCCGTATTTCGCGTAAGGAGGTCCATTATG \\
\hline no SD & TAATAATAACCTGGGCAGGCCATGTCTGCCCGTATTTCGCGTAACCTCCTCCATTATG \\
\hline oSD & TAATAATAACCTGGGCAGGCCATGTCTGCCCGTATTTCGCGCACCACAATCCATTATG \\
\hline oSD without SL & $\begin{array}{l}\text { TAATAATAACCTGGGCAGGCCATGCCCGGGTCTGCCCGTATTTCGCGCACCACAATCCAT } \\
\text { TATG }\end{array}$ \\
\hline
\end{tabular}

For frameshifting sites, the slippery sequence is underlined. In-frame control sequences corresponding to each target sequence are indicated by 'if'. For -1 frameshifting, a $\mathrm{C}$ was added within the slippery sequence; for +1 frameshifting, the $\mathrm{T}$ of the TGA stop codon was removed; and for stop codon readthrough, the stop codon was replaced by AAG. The dnaX and prfB frameshifting sites were also obtained with a specific CACCAC oRibo-T SD sequence (called oSD) upstream from the slippery sequence (dnaXoSD and prfBoSD, respectively). The SmaI site used for stem-loop (SL) cloning is indicated in bold typeface. To allow translation of the F-luc gene after frameshifting, the UGA stop codon in the -1 frame of the dnaX frameshifting site was changed to an UGU codon.

cOmplete Protease Inhibitor Cocktail (Roche)) with $50 \mu 1$ of acid-washed glass beads (Sigma) (8). Cells were lysed by vortexing for $30 \mathrm{~min}$ at $4{ }^{\circ} \mathrm{C}$. Luciferase and $\beta$-galactosidase activities were quantified, in $1-10 \mu l$ and $20 \mu$ l of cell extract, respectively (8). $\beta$-Galactosidase activity was measured through the hydrolysis of ONPG by determination of optical density at $420 \mathrm{~nm}$. Luciferase activity was measured with a Tecan Infinite M200 pro luminometer for light detection after addition of luciferin $0,2 \mathrm{mM}$ and ATP $2 \mathrm{mM}$ in luc buffer.

The translational efficiency of the ribosomes was calculated as the ratio of luciferase activity to $\beta$-galactosidase activity for the target, divided by the ratio of luciferase activity to $\beta$-galactosidase activity for the corresponding in-frame construct. We calculated the median value for at least six independent experiments. The significance of differences was determined in Student's $t$-tests.

We used constructs with a lac $Z$ gene carrying a wild-type SD sequence AGGAGG to analyze the translational efficiency of wild-type ribosomes. For assessments of the translational efficiency of oRibo-T ribosomes, we used the corresponding constructs with a mutated oSD sequence CACCAC upstream from the lac $Z$ gene, in the presence of the vector encoding the oRibo-T ribosomes. The mutated oSD sequence was complementary to the $3^{\prime}$ end of the $16 \mathrm{~S}$ ribosomal RNA of the oRibo-T ribosomes. In strains containing this vector, both wild-type and oRibo-T ribosomes are present, but only the oRibo-T ribosomes can initiate lac $Z$ translation, because of the mutated oSD sequence (see below).

\section{Total RNA purification}

Exponential cultures of the BL21 and BL21 carrying the poRibo-T2 vector cells were mechanically broken with beads and total RNA extracted following the QIAGEN RNeasy, Macherey-Nalgen Nucleospin RNA, and Nucleospin RNA Plus kit protocols. Following the RNA Easy protocol, a DNase treatment was done according to the QIAGEN RNase-free DNase Set.

\section{RESULTS}

\section{Validation of the orthogonal system}

The presence of the oRibo-T ribosomes in the cells was controlled by expression of the oRibo-T hybrid 16S/23S rRNA molecule, in addition to the individual chromosomal rRNA molecules (Figure 1B). Crosstalk between wild-type ribosomes and the orthogonal mRNA has recently been described (9). We checked the level of crosstalk in our system and found that it was too low to affect the results (Figure 1C). Indeed, in the presence of wild-type ribosomes only, constructs with a mutated oSD sequence upstream from the lac $Z$ gene generate too little $\beta$-galactosidase activity for measurement in our conditions (5-fold less $\beta$-galactosidase activity). Similarly, expression of the luc gene with the mutated oSD sequence resulted in levels of activity one tenth those observed with oRibo-T ribosomes (see also below).

With our validated system, we first analyzed the range of translational missense error rates done by the oRibo-T ribosomes in reading near-cognate codons (amino acid mis- 
A
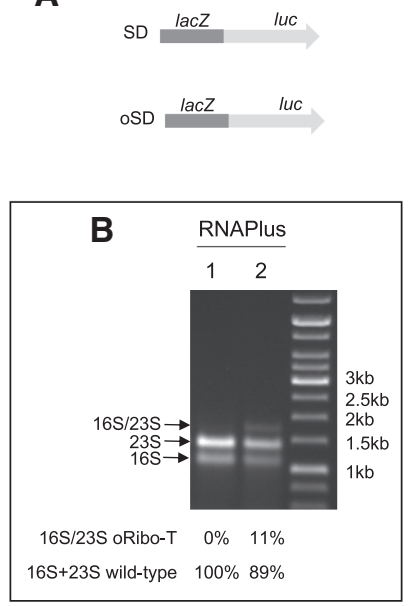
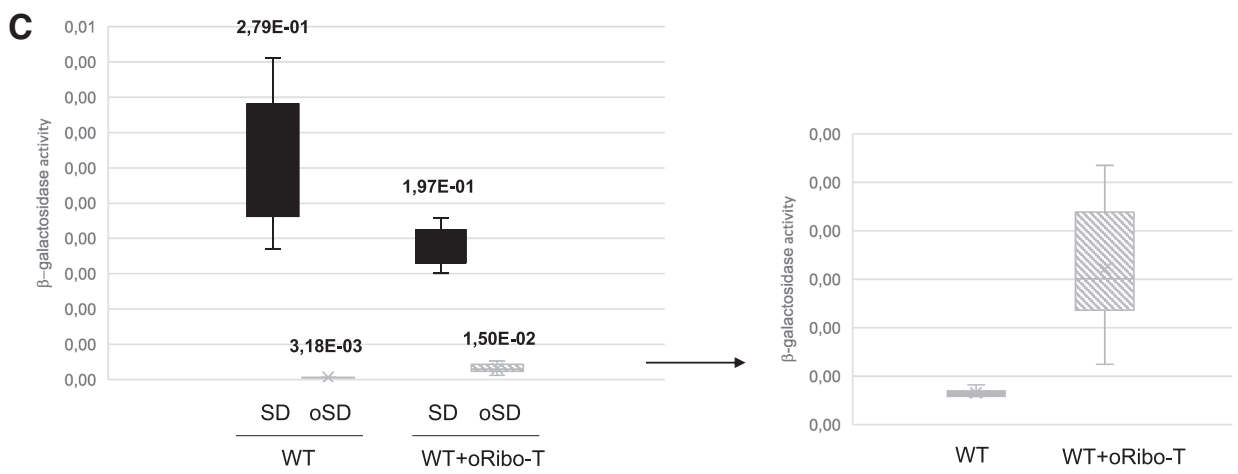

Figure 1. Translation of $l a c Z$ with either the wt SD or the oSD sequences by the wild-type or oRiboT ribosomes. The constructs analyzed are shown on the left (A). Agarose gel electrophoresis of total RNA preparation from cells expressing either the wild-type ribosomes (1 (BL21 cells)) or the wild-type and oRiboT ribosomes (2 (BL21 + poRibo-T2 vector cells)). Results obtained with the RNAPlus kit are shown with, below, the quantification of the respective rRNA species (B). The $\beta$-galactosidase activities with arbitrary units are shown for the wt SD lac $Z$ (SD, black) or the oSD lac $Z$ (oSD, light gray) translated by wild-type (WT), or oRibo-T (oSD, hatched light gray) ribosomes. Translation of the oSD lac Z results (light gray and hatched light gray) are shown on a smaller scale on the right panel indicated by an arrow (C). Results are from at least six independent experiments, with the median value indicated

incorporation). To this aim we used the same approach than the one developed by Farabaugh's group. It relies onto the modification of an essential lysine in the active site of firefly luciferase (10). Misincorporation of the wild-type aminoacid at the mutant codon restore enzymatic activity. The misreading frequency was shown to reflect the details of codon:anticodon pairing, as well as how cognate and nearcognate aa-tRNA compete for the codon (10).

\section{Ribo-T ribosomes discriminate tRNAs as efficiently as WT ribosomes}

We used a reporter system that measures the frequency of near-cognate misreading errors by Lys-tRNA ${ }^{\text {UUU }}$ at K529 position in the luciferase gene. As previously shown by Farabaugh's group mutant proteins retaining activity result from mistranslation of the mutant codon to incorporate lysine at position 529 (10).

We tested the near-cognate misreading errors with codons differing from AAA lysine codon by a single nucleotide in the first (CAA), second (AGA) and third (AAT) position. In each case, the Lys-tRNA ${ }^{U U U}$ can recognize the mutant codon as a near-cognate substrate with only one base mismatch. We also replaced the AAA lysine codon with a TTT phenylalanine codon, a negative control for misreading as no base pairs can be formed with the LystRNA ${ }^{U U U}$. The luciferase is expressed as a single polypeptide with the upstream $\beta$-galactosidase. Any difference in the ratio of the luciferase activity to the $\beta$-galactosidase activity must reflect a change in the activity of the mutant luciferase.

We measured the enzyme activities for each mutant construct translated by wild-type or oRibo-T ribosomes (Figure 2). Whatever the mutated codon was, results were similar between the wild-type and oRibo-T ribosomes ( $P$-value of 0.807 for TTT, 0.51 for CAA, 0.402 for AGA and 0.519 for AAT; $P$-value of 0.284 for the wild-type AAA). This in- dicated the two ribosomes display the same frequency of translational misreading errors. Interestingly the range of misincorporation is the same than previously observed (10), with a higher level at AAT, than AGA or CAA indicating than oRibo-T successfully discriminates the wobble position from the two other positions.

\section{Ribo-T ribosomes are prone to frameshift independently of the $\mathrm{SD}$ sequence}

We then investigated the way in which oRibo-T ribosomes deal with programmed frameshifting sequences. In essence, frameshifting is triggered by two features: a slippery sequence favoring tRNA slippage and one or several stimulatory elements that enhance the process by inducing a ribosomal pause.

We selected three programmed -1 frameshifting sites from the IS911 and IS3 insertion sequence elements and the E. coli dnaX gene (Figure 3) (11). Frameshifting is stimulated by two elements: an RNA structure (a stem-loop for IS911 and $d n a X$, a pseudoknot for IS3) located downstream from the slippery site, and an SD sequence located upstream from the slippery site. The combination of these elements, together with the highly efficient slippery sequence, gave a -1 frameshifting efficiency of about $13 \%, 8 \%$ and $60 \%$ for IS911, IS3 and dnaX, respectively (12-14).

We first measured frameshifting efficiencies with WT sequences (i.e. with a wt SD). As oRibo-T ribosomes cannot base pair to the WT SD, we assumed that they would be unable to frameshift. Surprisingly, this turned out not to be the case, as the efficiency of -1 ribosomal frameshifting was $12 \%$ for IS911 with wild-type ribosomes and $13 \%$ with oRibo-T ribosomes ( $P$-value of 0.8 ), $29 \%$ for IS3 with wild-type ribosomes and $34 \%$ with oRiboT ribosomes ( $P$-value of 0.97$)$, $45 \%$ for dnaX with either type of ribosomes $(P$-value of 0.77 ) (Figure 4A). These results suggest that oRibo-T ribosomes frameshift independently of the SD. We investigated 
A

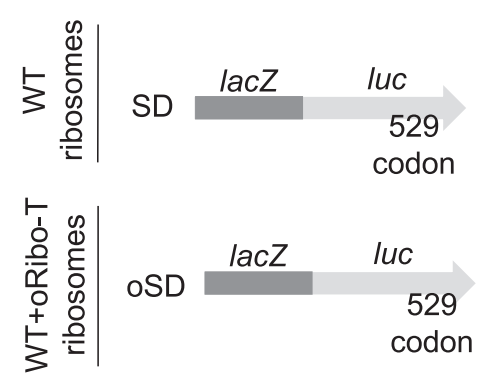

C
B

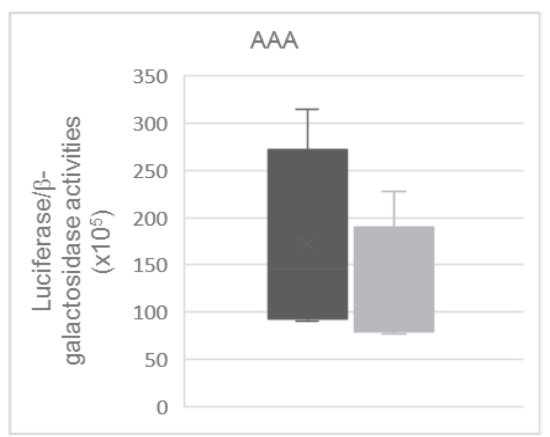

WT oRibo-T

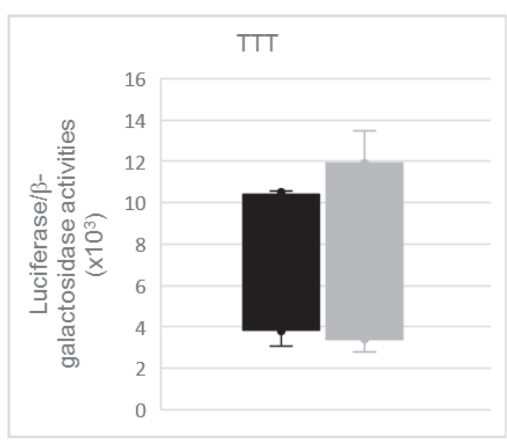

WT oRibo-T

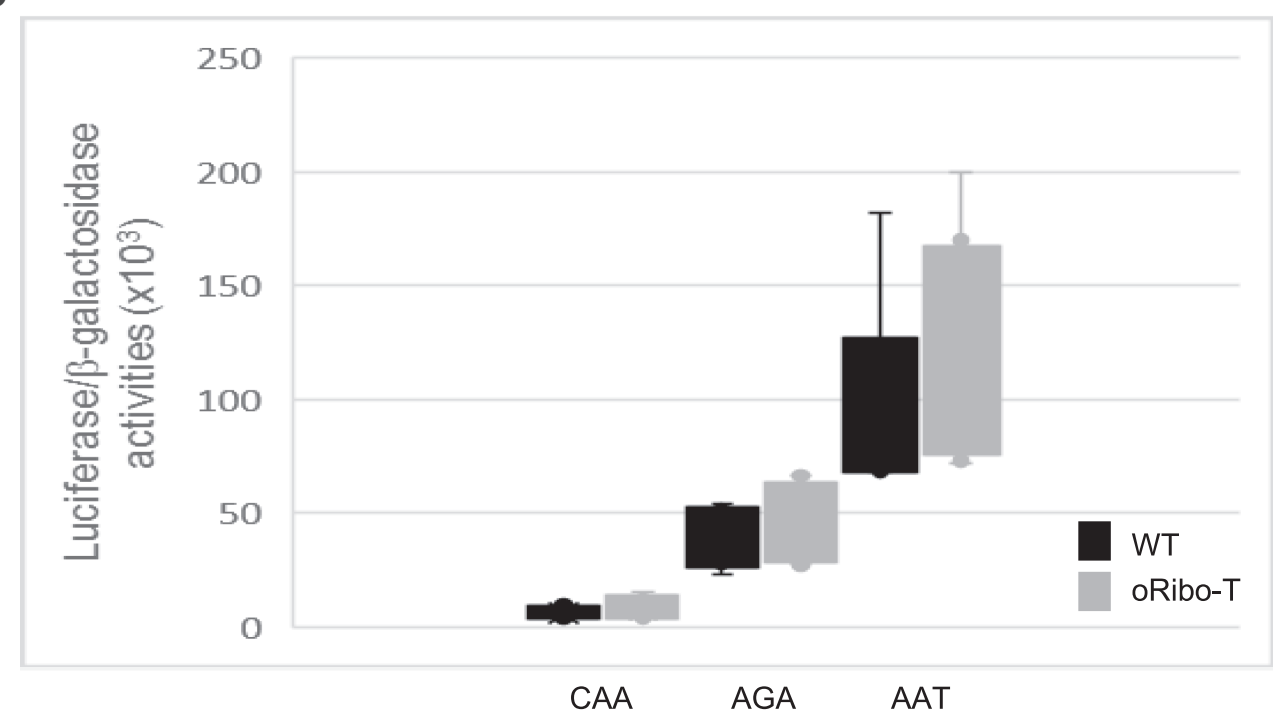

Figure 2. Translational misreading errors. The ratio of luciferase activity to $\beta$-galactosidase activity is shown as arbitrary units for each construct carrying the indicated sequence of the codon 529 of the luciferase gene (wild-type AAA, or mutants TTT, CAA, AGA and AAT). The positive control (wild-type AAA) and the negative control (mutant TTT with no luciferase activity) are shown in (B), the CAA, AGA and AAT mutants in (C). The constructs analyzed are shown in (A), with the type of ribosomes present in the cells. Translations of the lacZ-luc fusion gene by wild-type (WT) (dark gray), or oRibo-T (oRibo-T) (light gray) ribosomes. Results are from at least six independent experiments.

this possibility by performing the reverse experiment. We replaced the dnaX SD sequence with an oSD recognized by oRibo-T ribosomes (dnaXoSD). This modification greatly decreased the frameshifting efficiency of WT ribosomes $(8 \%$ versus $45 \%$; $P$-value of $\left.1.7 \times 10^{-3}\right)$, but this change had no significant impact on oRibo-T ribosome frameshifting ( $45 \%$ versus $48 \% ; P$-value 0.85 ) (Figure $4 \mathrm{~B}$ ), confirming that oRibo-T ribosomes are insensitive to the presence of an $\mathrm{SD}$ sequence even though they maintain high levels of -1 frameshifting.

This surprising result led us to investigate whether this effect was specific to -1 frameshifting signals, or also applied to +1 frameshifting.

For +1 frameshifting, we selected the site from the E. coli $\operatorname{prfB}$ gene (Figure 5A). Frameshifting efficiency is typically around $30-50 \%$, and this process is dependent on a poor termination context of the stop codon (UGA C), together with the stimulatory effect of an SD sequence located upstream from the slippery site (15).
We obtained a +1 frameshifting efficiency of $34 \%$ for the WT ribosome, confirming previous results (16). However, oRibo-T ribosomes frameshift inefficiently on this sequence, with an efficiency of only $2 \%$ ( $P$-value of 8.9 $\times 10^{-9}$ versus wild-type ribosomes; Figure 5B). This may be because the stimulatory SD sequence is wild-type and is not, therefore, recognized by oRibo-T ribosomes. We addressed this possibility, by replacing the wild-type SD sequence with a oSD sequence recognized by oRibo-T ribosomes. Interestingly, this did not restore the +1 frameshifting efficiency of oRibo-T ribosomes $(1.1 \%, P$-value of 0.56 relative to the previous finding), but it did strongly decrease the +1 frameshifting activity of wild-type ribosomes $\left(0.3 \%\right.$ versus $34 \%, P$-value of $\left.1.6 \times 10^{-5}\right)$, which were unable to recognize this oSD sequence as an SD sequence (Figure 5C).

These results confirm that oRibo-T ribosomes are unable to sense $\mathrm{SD}$ sequences and use them as stimulatory elements for frameshifting to either the -1 or +1 frame. 


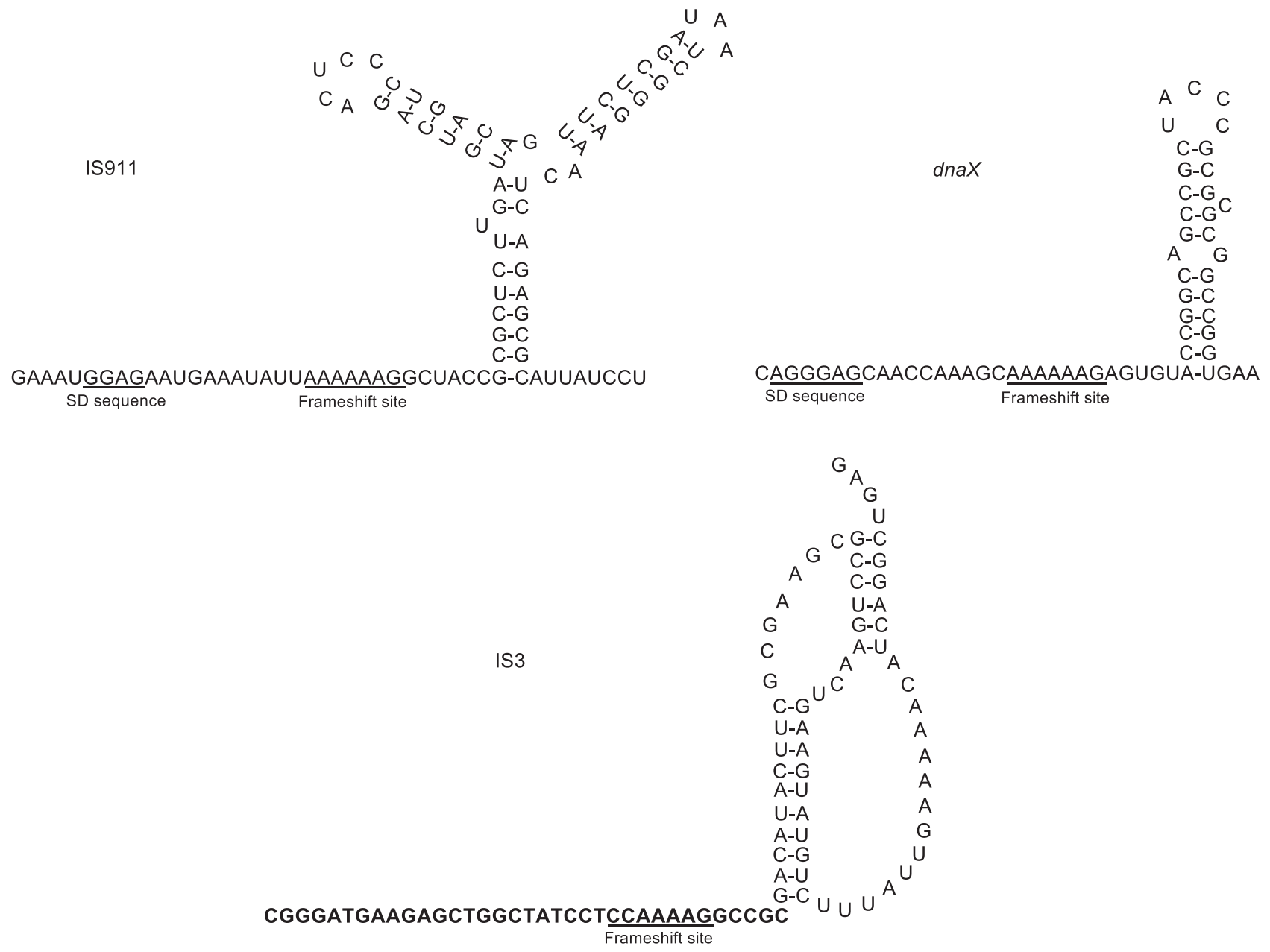

Figure 3. The -1 frameshifting sites. For each sequence the slippery sequence and the SD are underlined. Two frameshifting sites come from IS elements, and one from the cellular dnaX gene. IS911, dnaX and IS3 frameshifting sites use a single or double stem loop or a pseudoknot respectively as stimulatory elements.

\section{Ribo-T ribosomes make inefficient use of the SD sequence to initiate translation}

Our data demonstrate the inability of oRibo-T ribosomes to use $\mathrm{SD}$ sequences to stimulate frameshifting. This led us to investigate the recognition of the SD sequence during translation initiation, when this sequence is used to assemble the ribosome at the start codon. We used the 46-nucleotide intergenic E. coli lacZ-lac $Y$ sequence, which carries the SD sequence promoting initiation at the lac $Y$ start codon to address this question. We modified this sequence by deleting the SD sequence or replacing it with the oSD sequence recognized only by oRibo-T ribosomes (see Materials and Methods, Table 1). In this system, lac $Z$ could be translated only by wild-type ribosomes, because all constructs carried a wild-type SD sequence upstream from lacZ. The ability of wild-type or oRibo-T ribosomes to translate $l u c$ depended on whether the second SD sequence was recognized.

In the absence of oRibo-T ribosomes, we observed almost no luciferase activity when the SD sequence upstream from the luc gene was removed. By contrast, when the wild-type SD sequence was used, we obtained high levels of luciferase activity, demonstrating the correct reconstitution of the SD dependence of translation initiation context by the reporter system (Figure 6A). In the presence of the oSD sequence, we observed no statistically significant difference with the absence of a SD sequence ( $P$-value 0.04 , versus no SD) in luciferase activity, which suggests that wild-type ribosomes cannot use this alternative oSD sequence to initiate translation (Figure 6B). Interestingly, in the presence of oRibo-T ribosomes, luciferase induction was increased by a factor of four $\left(P\right.$-value $\left.4.7 \times 10^{-4}\right)$, indicating that oRibo-T ribosomes can use this alternative oSD sequence, albeit inefficiently relative to WT ribosomes in the presence of a wildtype SD sequence (Figure 6B).

In prokaryotes, ribosomes are thought to initiate the translation of a downstream gene either by $70 \mathrm{~S}$ re-initiation after translation of the upstream gene is terminated, or by internal loading of the $30 \mathrm{~S}$ at the initiation site $(70 \mathrm{~S}$ for Ribo-T with tethered subunits) $(17,18)$. Both these processes depend on the distance between the upstream stop codon and the downstream start codon, with a seven-codon interval thought to be crucial for the posttermination reinitiation of protein synthesis by ribosomes. The long intergenic sequence between $l a c Z$ and $l u c$, with three successive 
A
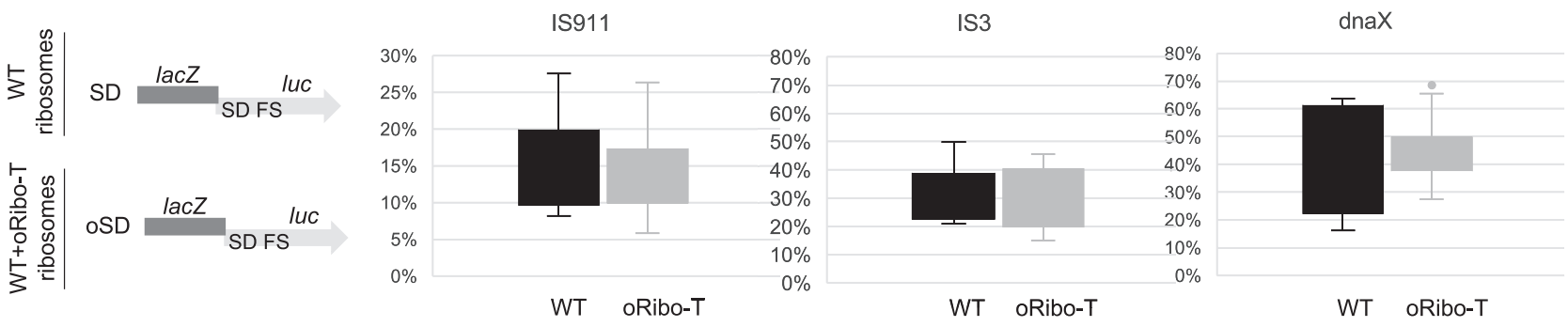

B
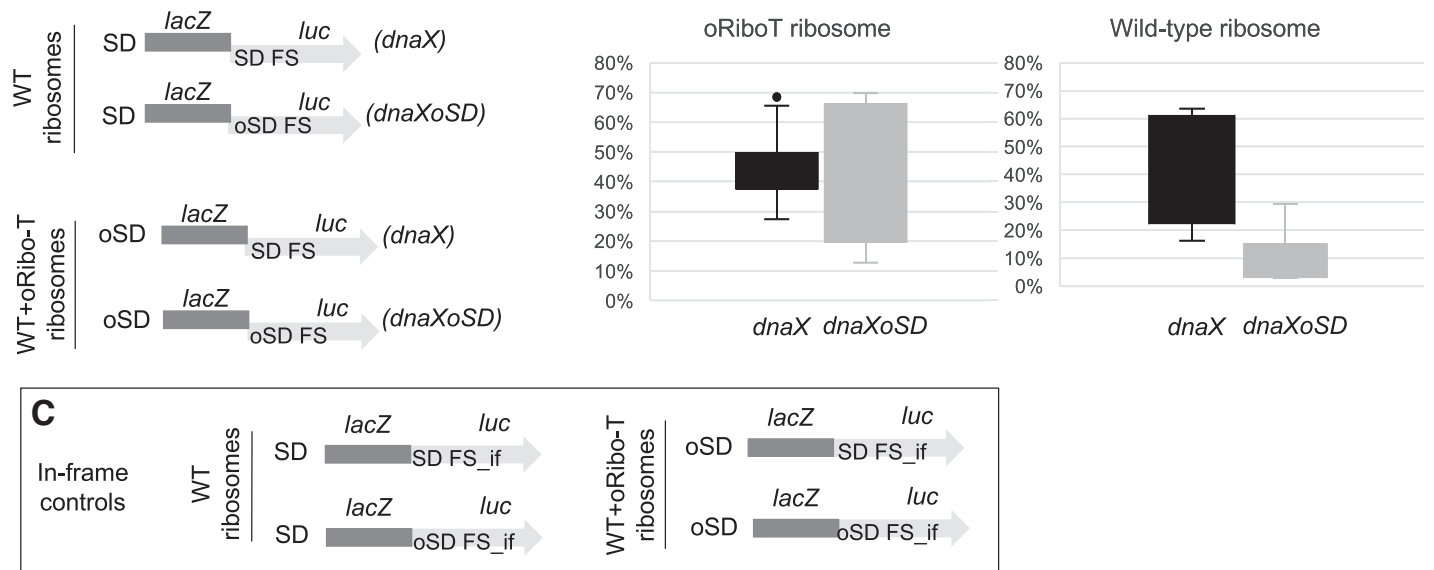

Figure 4. Translational efficiencies during -1 frameshifting. The constructs analyzed are shown on the left, with the type of ribosomes present in the cells, and in-frame controls in (C). The percent frameshifting is shown for the IS911, IS3 and dnaX sequences translated by wild-type (WT) (dark gray) and oRibo-T (light gray) ribosomes (A); and for the $\operatorname{dnaX}$ (dark gray) and dnaXoSD (light gray) sequences translated by oRibo-T or wild-type ribosomes (B). Results are from at least six independent experiments.

stop codons ending the first ORF, would not favor a scanning process.

We investigated whether the type of ribosomes translating lac $Z$ influenced the rate of translation initiation for the $l u c$ gene, by replacing the wild-type $\mathrm{SD}$ sequence upstream from $l a c Z$ with a oSD sequence. We reasoned that if $70 \mathrm{~S}$ reinitiation could occur, we would observe an increase in luciferase activity due to oRibo-T ribosomes initiating translation upstream in lac $Z$. We found that expression of the luciferase reporter gene was four times stronger when the oSD sequence was placed in front of the start codon for the upstream lac $Z$ than in the total absence of an SD sequence (no-SD; $P$-value $9.8 \times 10^{-6} ;$ Figure $6 \mathrm{C}$ ). This increase was of a similar magnitude to that obtained when a wild-type SD sequence was placed upstream from the $l a c Z$ gene (Figure $6 \mathrm{~B})$. The higher activity ratio obtained for the oRibo-T ribosomes translating both lac $Z$ and $l u c$ (Figure $6 \mathrm{C}$ ) resulted from lower levels of $\beta$-galactosidase activity than were obtained when wild-type ribosomes translated lac Z (oSD data in Figure 6B (2)). Thus, translation initiation at the luc gene is not dependent on the nature of the ribosomes translating the upstream lac $Z$ gene. For confirmation of this observation, we inserted a stable stem loop structure immediately downstream from $l a c Z$, to act as a roadblock for potential scanning ribosomes. We found that structure had no effect on the initiation of $l u c$ translation ( $P$-value 0.22$)$, strongly suggesting that the initiation of luc translation occurred through the internal loading of oRibo-T ribosomes (Figure 6D). Overall, our results indicate that oRibo-T ri- bosomes do not use SD sequence efficiently during either initiation or programmed frameshifting.

\section{Ribo-T ribosomes terminate translation less efficiently at stop codons}

Stop codon readthrough occurs when the ribosome reads the termination codon as a sense codon and continues translation until the next stop codon in the same reading frame (19). Quantifying stop codon readthrough is an efficient way to assess the accuracy of ribosomes and their ability to accommodate near-cognate tRNAs. Unfortunately, no natural stop codon readthrough sequence has ever been identified in E. coli. We therefore used the stop codon sequence of the $m t m B l$ gene encoding monomethylamine methyltransferase from the archaea Methanosarcina barkeri (20), which allows the insertion of a pyrrolysine, to estimate the accuracy of oRibo-T ribosomes during translation termination. This sequence has been shown to promote stop codon readthrough in E. coli, even in the absence of pyrrolysine (7).

We found that oRibo-T ribosomes terminated translation less efficiently than wild-type ribosomes (Figure 7; 0.06\% versus $0.01 \%, P$-value of $4.7 \times 10^{-7} ; 0.27 \%$ versus $0.10 \%$, $P$-value of $5 \times 10^{-4} ; 0.04 \%$ versus $0.01 \%, P$-value of $2.5 \times$ $10^{-4}$, for UAG, UGA and UAA stop codons, respectively). Thus, oRibo-T ribosomes display a slight impairment of stop codon recognition. 
A

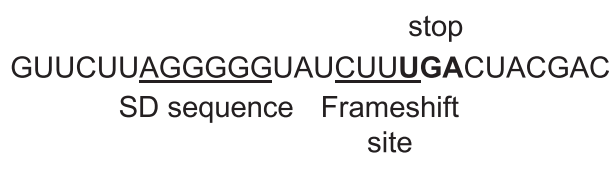

B

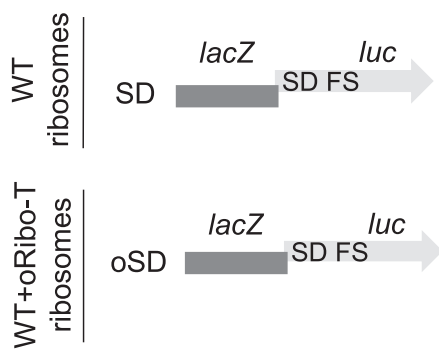

C

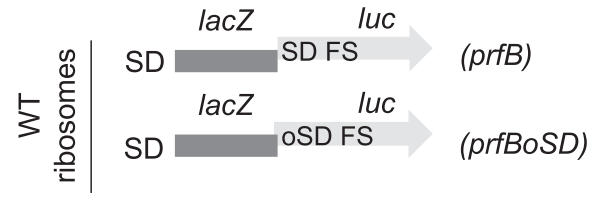
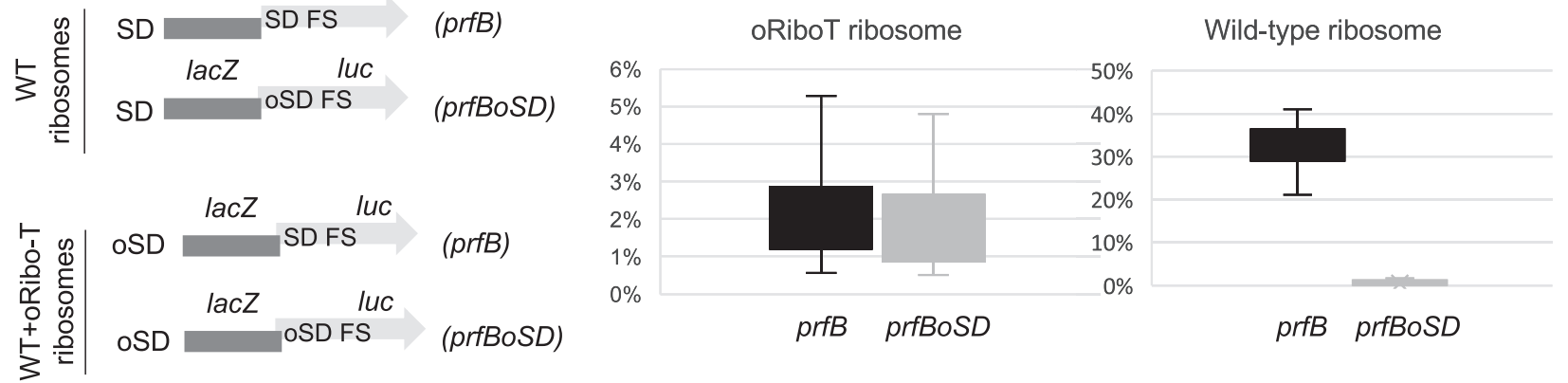

\begin{tabular}{|c|c|c|c|c|c|c|c|c|}
\hline \multirow{3}{*}{$\begin{array}{l}\text { D } \\
\text { In-frame } \\
\text { controls }\end{array}$} & \multirow{3}{*}{ 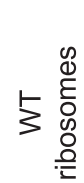 } & & \multirow[t]{2}{*}{ lacZ } & \multirow[t]{2}{*}{ luc } & \multirow{3}{*}{ 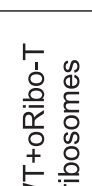 } & \multirow[b]{2}{*}{ OSD } & lacZ & luc \\
\hline & & SD & & & & & & $=S \_$if \\
\hline & & SD & lacZ & $\begin{array}{l}\text { luc } \\
\text { FS if }\end{array}$ & & & $l a c Z$ & $\begin{array}{c}\text { luc } \\
\text { FS_if }\end{array}$ \\
\hline
\end{tabular}

Figure 5. $p r f B+1$ frameshifting. Sequence of the $E$. coli frameshifting site (A). Translational efficiencies for $p r f B$ frameshifting, for wild-type ribosomes (dark gray) and oRibo-T ribosomes (light gray) (B); or for the $\operatorname{prfB}$ (dark gray) and $\operatorname{prfBoSD}$ (light gray) sequences translated by oRibo-T or wild-type ribosomes $(\mathbf{C})$. The constructs analyzed are shown on the left, with the type of ribosomes present in the cells, and in-frame controls in (D). Results are from at least six independent experiments.

\section{DISCUSSION}

In all organisms, the ribosome consists of two unequal subunits, the large and small ribosomal subunits, which associate with each other in a labile manner, via bridges. It remains unclear why the ribosome consists of two loosely associated subunits that must be associated for translocation to occur. It has been suggested that the mutual mobility of the two ribosomal subunits is essential for the translocation mechanism. However, a functional ribosome with covalently linked subunits has been successfully created independently by two groups $(2,21)$, calling this hypothesis into question, or suggesting that the covalently linked structure retained some mobility.

Surprisingly, tethered ribosomes (also called Ribo-T ribosomes) support cell growth and are not associated with any obvious phenotype, other than a slow-growth phenotype that has been attributed to slower rRNA processing rather than translational defects (3). We investigated the translational accuracy of oRibo-T ribosomes. For this purpose, we constructed reporters for measuring translational errors such as amino acid misincorporation, stop codon readthrough, and frameshifting. Such recoding events con- stitute useful tools for studying translation accuracy, as the sequences concerned alter ribosome proofreading activity, and translational defects are amplified at these sites. During translation, the oRibo-T ribosomes show the expected misreading error rates of near-cognate codons compared to the wild-type ribosomes, whatever is the mismatch position (Figure 2). This highlights that the ability of Ribo-T ribosomes to discriminate between cognate and near-cognate or non-cognate tRNAs is not impacted by tethering both subunits. To our surprise, we observed no change in -1 frameshifting efficiency, whatever the type of SD sequence (either wild-type or oSD) used (Figure 4B). These initial results were confirmed for +1 frameshifting (Figure 5C). Interestingly, the efficiency of +1 frameshifting was much lower for oRibo- $\mathrm{T}$ than for wild-type ribosomes (Figure 5B). These results suggest that oRibo-T ribosomes do not use SD sequences to promote frameshifting. The tether may intrinsically stimulate frameshifting so that the presence or absence of the SD has no further effect on its efficiency. The tether is epistatic to the SD-ASD interaction, in a way that support regular $(-1$ event) or poor frameshifting $(+1$ event). This may reflect the different roles of SD-ASD in- 
A

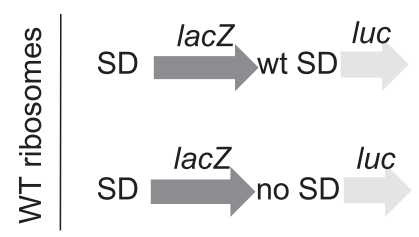

B

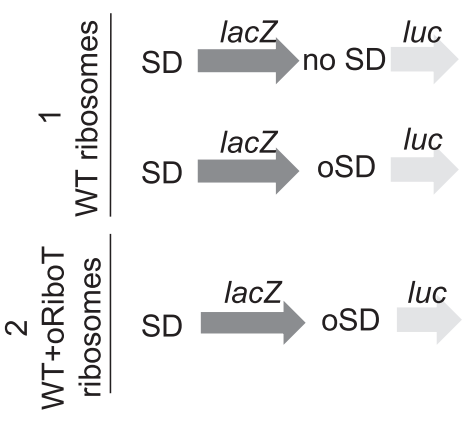

C
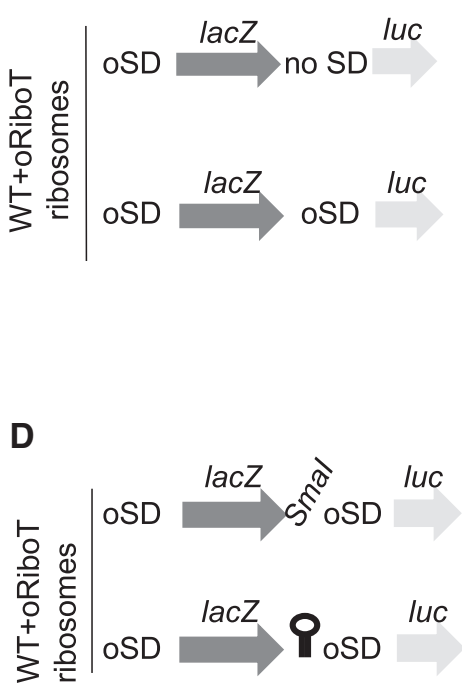

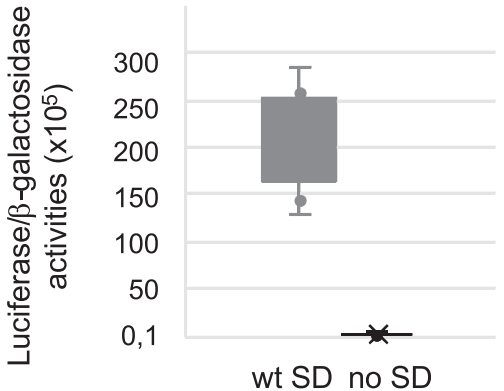

wt SD no SD

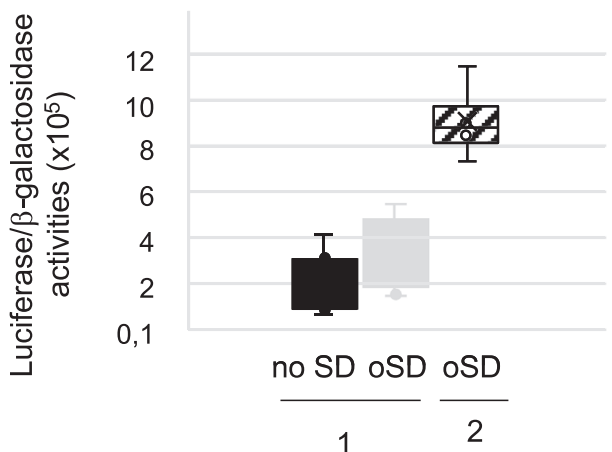

1

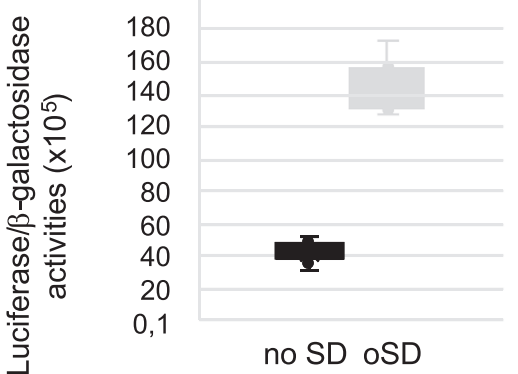

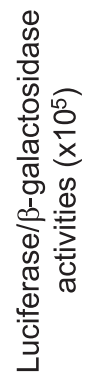

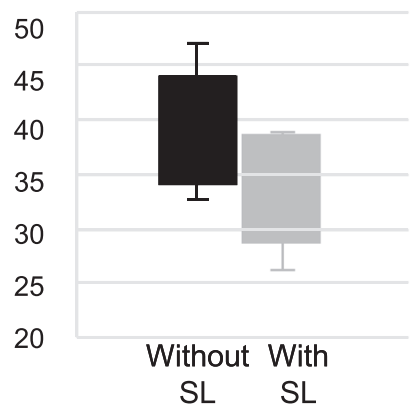

Figure 6. Internal initiation of wild-type ribosomes. The constructs analyzed are shown on the left, with the type of ribosomes present in the cells. The ratio of luciferase activity to $\beta$-galactosidase activity measured for each construct is shown in the right box plot, as arbitrary units. Translations of the lac Z gene by wild-type ribosomes (WT), and of the luc gene with either a wild-type SD (wt SD) (dark gray) or no SD (black) by wild-type ribosomes (A). Translations of lac $Z$ by wild-type ribosomes, and of $l u c$ with either no SD (black) or a oSD (light gray) by wild-type ribosomes (1), or a oSD (hatched) by wild-type and oRibo-T ribosomes (2) (B). Translations of lac $Z$ by oRibo-T ribosomes and of luc with either no SD (black) or a oSD (light gray) by wild-type and oRibo-T ribosomes (C), or by oRibo-T ribosomes with (light gray) or without (black) a stem-loop structure upstream from luc (D). The SmaI site specific of the sequence without a stem-loop structure is indicated. Results are from at least six independent experiments. 

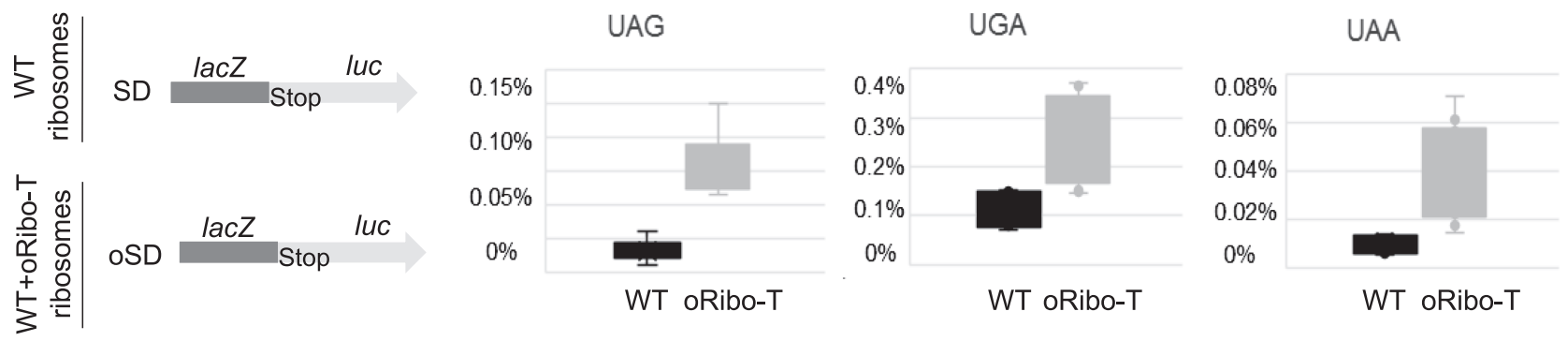

Figure 7. Translational readthrough efficiencies. The percent readthrough is shown for the three stop codons encountered by the wild-type (WT) (dark gray) and oRibo-T (light gray) ribosomes. The constructs analyzed are shown on the left, with the type of ribosome present in the cells. Results are from at least six independent experiments.

teraction in these two events. Indeed, for +1 frameshifting, this interaction causes the release of the deacylated Esite tRNA, destabilizing the ribosome complex and affecting P-site tRNA slippage $(22,23)$, while for -1 frameshifting the $\mathrm{SD}$ is thought to slow-down the translocation step to increase the probably to tRNAs to slip in the -1 reading frame. Both types of frameshifting are known to involve base pairing between the SD sequence and the ASD sequence on the 16S rRNA to increase frameshifting efficiency $(22,24-27)$.

We therefore propose that in oRibo-T the physical link between both subunits precludes the translocation from taking place normally. In this situation, as the translocation is already slowed down, the SD no longer has the stimulating role that it plays in WT condition. Although, this situation is atypical, because frameshifting occurs during the elongation phase and SD sequences are usually used at the initiation step. This may reflect a general trend towards oRibo-T ribosomes not efficiently using SD sequences for either elongation or initiation. We therefore assessed the ability of oRibo-T ribosomes to use SD sequences during translation initiation. We constructed a specific dual reporter for monitoring translation efficiency in the presence of various SD sequences. We found that oRibo-T ribosomes used the SD sequence much less efficiently than wild-type ribosomes (Figure 6).

The translation initiation rate of a given gene can be modulated by the structural accessibility of the SD sequence, the thermodynamic binding potential between the SD sequence and the ASD sequence, and the exact positioning of the SD sequence relative to the start codon. Stronger SD-ASD sequence interactions are associated with a higher translation efficiency, as they stabilize the assembly of the translation initiation complex. Hockenberry et al. (28) suggested that decreasing the translation initiation rates for a large number of genes would probably lead to substantially longer doubling times. This may be the case for $E$. coli cells with oRibo-T ribosomes, which had longer doubling times and a lower translational efficiency, with a protein synthesis rate $50 \%$ that in cells with wild-type ribosomes (2). Thus the slower growth rate of the Ribo-T expressing strain may be due to both defects in ribosome biogenesis and in translation. In recent years, the role of SD sequence as the most important element governing various aspects of translation initiation (efficiency, reading frame selection, regulation) has been called into question. The existence of mRNAs de- void of the SD sequence clearly indicated that this sequence was neither necessary nor sufficient for translation initiation (29). A local absence of RNA secondary structure has been shown to be necessary and sufficient for the initiation of SD sequence-independent translation (30) although the presence of the SD sequence greatly increased the efficiency of translation initiation (31).

The mechanism of translation initiation by oRibo-T ribosomes remains unclear. However, the two subunits are linked together via helix 44 (h44) in these ribosomes (2). This helix is known to play an important role in the fidelity of translation initiation (32). The tethering of ribosomes may allow sufficient translation for cell viability, but it probably also has structural consequences for the geometry of h44. The ASD sequence is located at the top of h44. We therefore suggest that its orientation is modified in oRibo$\mathrm{T}$ ribosomes, preventing them from efficiently using SD sequences in a normal manner during translation initiation and elongation. The elucidation of a high-resolution structure for Ribo-T ribosomes would help to resolve this question.

We also observed that oRibo-T ribosomes promoted termination less efficiently than wild-type ribosomes (Figure 7). The termination and stop codon readthrough processes are in competition every time a ribosome encounters a stop codon. The balance between these processes is displaced toward readthrough when the ribosomal A-site is occupied by a near-cognate tRNA. Not only does efficient translation termination require the coordinated action of release factors, it also depends on the conformational dynamics of the factors and the ribosome. One may suggest that key conformational motions of the ribosome during termination, may be less dynamic, and associated with an A-site less able to bind termination factors in Ribo-T ribosomes.

These results are consistent with a previous ribosome profiling analysis in Ribo-T-expressing cells, which highlighted an increase in ribosome density at start codons and close to the $3^{\prime}$ ends of genes (3). The tethering of the ribosomal subunits had mild effects on the initiation and termination/recycling steps of translation.

Our work reveals the specific behavior of tethered ribosomes and highlights the greater-than-expected flexibility of ribosomes. Undoubtedly, pushing ribosomes to their limits will also provide important information about their functioning. One question of interest concerns whether tethered ribosomes would be efficient in eukaryotes, which have a 
different mechanism of translation initiation not involving SD-ASD interaction.

\section{DATA AVAILABILITY}

Data and plasmids available upon request.

\section{ACKNOWLEDGEMENTS}

The English of this manuscript was corrected by Alex Edelman \& Associates. We would like to thank Shura Mankin for providing the strains and plasmids, sharing unpublished results and for his constructive comments about the manuscript.

\section{FUNDING}

ANR rescue_ribosome Grant [ANR-17-CE12-0024-01]. Funding for open access charge: Agence Nationale de la Recherche [ANR-17-CE12-0024-01].

Conflict of interest statement. None declared.

\section{REFERENCES}

1. Rodnina,M.V. (2018) Translation in prokaryotes. Cold Spring Harb. Perspect. Biol., 10, a032664.

2. Orelle,C., Carlson,E.D., Szal,T., Florin,T., Jewett,M.C. and Mankin,A.S. (2015) Protein synthesis by ribosomes with tethered subunits. Nature, 524, 119-124.

3. Aleksashin,N.A., Leppik,M., Hockenberry,A.J., Klepacki,D., Vazquez-Laslop,N., Jewett,M.C., Remme,J. and Mankin,A.S. (2019) Assembly and functionality of the ribosome with tethered subunits. Nat. Commun., 10, 930.

4. Rozov,A., Demeshkina,N., Westhof,E., Yusupov,M. and Yusupova,G. (2015) Structural insights into the translational infidelity mechanism. Nat. Commun., 6, 7251 .

5. Namy,O., Rousset,J.P., Napthine,S. and Brierley,I. (2004) Reprogrammed genetic decoding in cellular gene expression. Mol. Cell, 13, 157-168.

6. Rackham,O. and Chin,J.W. (2005) A network of orthogonal ribosome x mRNA pairs. Nat. Chem. Biol., 1, 159-166.

7. Namy,O., Zhou,Y., Gundllapalli,S., Polycarpo,C.R., Denise,A., Rousset,J.P., Soll,D. and Ambrogelly,A. (2007) Adding pyrrolysine to the Escherichia coli genetic code. FEBS Lett., 581, 5282-5288.

8. Stahl,G., Bidou,L., Rousset,J.P. and Cassan,M. (1995) Versatile vectors to study recoding: conservation of rules between yeast and mammalian cells. Nucleic Acids Res., 23, 1557-1560.

9. Carlson,E.D., d'Aquino,A.E., Kim,D.S., Fulk,E.M., Hoang,K., Szal,T., Mankin,A.S. and Jewett,M.C. (2019) Engineered ribosomes with tethered subunits for expanding biological function. Nat. Commun., 10, 3920

10. Kramer,E.B. and Farabaugh,P.J. (2007) The frequency of translational misreading errors in E. coli is largely determined by tRNA competition. RNA, 13,87-96.

11. Atkins,J.F., Loughran,G., Bhatt,P.R., Firth,A.E. and Baranov,P.V. (2016) Ribosomal frameshifting and transcriptional slippage: from genetic steganography and cryptography to adventitious use. Nucleic Acids Res., 44, 7007-7078.

12. Polard,P., Prere,M.F., Chandler,M. and Fayet,O. (1991) Programmed translational frameshifting and initiation at an AUU codon in gene expression of bacterial insertion sequence IS911. J. Mol. Biol., 222, 465-477.
13. Sekine,Y., Eisaki,N. and Ohtsubo,E. (1994) Translational control in production of transposase and in transposition of insertion sequence IS3. J. Mol. Biol., 235, 1406-1420.

14. Tsuchihashi,Z. and Brown,P.O. (1992) Sequence requirements for efficient translational frameshifting in the Escherichia coli dnaX gene and the role of an unstable interaction between tRNA(Lys) and an AAG lysine codon. Genes Dev., 6, 511-519.

15. Weiss,R.B., Dunn,D.M., Dahlberg,A.E., Atkins,J.F. and Gesteland,R.F. (1988) Reading frame switch caused by base-pair formation between the $3^{\prime}$ end of $16 \mathrm{~S}$ rRNA and the mRNA during elongation of protein synthesis in Escherichia coli. EMBO J., 7, $1503-1507$.

16. Curran,J.F. and Yarus,M. (1988) Use of tRNA suppressors to probe regulation of Escherichia coli release factor 2. J. Mol. Biol., 203, $75-83$.

17. Yamamoto,H., Wittek,D., Gupta,R., Qin,B., Ueda,T., Krause,R., Yamamoto,K., Albrecht,R., Pech,M. and Nierhaus,K.H. (2016) $70 \mathrm{~S}$-scanning initiation is a novel and frequent initiation mode of ribosomal translation in bacteria. Proc. Natl. Acad. Sci. U.S.A., 113, E1180-E1189.

18. Karamyshev,A.L., Karamysheva,Z.N., Yamami,T., Ito,K. and Nakamura,Y. (2004) Transient idling of posttermination ribosomes ready to reinitiate protein synthesis. Biochimie, 86, 933-938.

19. Dabrowski,M., Bukowy-Bieryllo,Z. and Zietkiewicz,E. (2015) Translational readthrough potential of natural termination codons in eucaryotes-The impact of RNA sequence. RNA Biol, 12, 950-958.

20. Burke,S.A., Lo,S.L. and Krzycki,J.A. (1998) Clustered genes encoding the methyltransferases of methanogenesis from monomethylamine. J. Bacteriol., 180, 3432-3440.

21. Fried,S.D., Schmied,W.H., Uttamapinant,C. and Chin,J.W. (2015) Ribosome subunit stapling for orthogonal translation in $E$. coli. Angew. Chem. Weinheim Bergstr. Ger., 127, 12982-12985.

22. Marquez,V., Wilson,D.N., Tate,W.P., Triana-Alonso,F. and Nierhaus, K.H. (2004) Maintaining the ribosomal reading frame: the influence of the E site during translational regulation of release factor 2. Cell, 118, 45-55.

23. Liao,P.Y., Gupta,P., Petrov,A.N., Dinman,J.D. and Lee,K.H. (2008) A new kinetic model reveals the synergistic effect of E-, P- and A-sites on +1 ribosomal frameshifting. Nucleic Acids Res. 36, 2619-2629.

24. Larsen,B., Wills,N.M., Gesteland,R.F. and Atkins,J.F. (1994) rRNA-mRNA base pairing stimulates a programmed -1 ribosomal frameshift. J. Bacteriol., 176, 6842-6851.

25. Baranov,P.V., Gesteland,R.F. and Atkins,J.F. (2002) Release factor 2 frameshifting sites in different bacteria. EMBO Rep., 3, 373-377.

26. Prere,M.F., Canal,I., Wills,N.M., Atkins,J.F. and Fayet,O. (2011) The interplay of mRNA stimulatory signals required for AUU-mediated initiation and programmed -1 ribosomal frameshifting in decoding of transposable element IS911. J. Bacteriol., 193, 2735-2744.

27. Kim,H.K. and Tinoco,I. Jr (2017) EF-G catalyzed translocation dynamics in the presence of ribosomal frameshifting stimulatory signals. Nucleic Acids Res., 45, 2865-2874.

28. Hockenberry,A.J., Stern,A.J., Amaral,L.A.N. and Jewett,M.C. (2018) Diversity of translation initiation mechanisms across bacterial species is driven by environmental conditions and growth demands. Mol. Biol. Evol., 35, 582-592.

29. Chang,B., Halgamuge,S. and Tang,S.L. (2006) Analysis of SD sequences in completed microbial genomes: non-SD-led genes are as common as SD-led genes. Gene, $\mathbf{3 7 3}, 90-99$.

30. Scharff,L.B., Childs,L., Walther,D. and Bock,R. (2011) Local absence of secondary structure permits translation of mRNAs that lack ribosome-binding sites. PLoS Genet., 7, e1002155.

31. Saito,K., Green,R. and Buskirk,A.R. (2020) Translational initiation in $E$. coli occurs at the correct sites genome-wide in the absence of mRNA-rRNA base-pairing. Elife, 9 , e55002.

32. Qin,D., Liu,Q., Devaraj,A. and Fredrick,K. (2012) Role of helix 44 of 16S rRNA in the fidelity of translation initiation. RNA, 18, 485-495. 